\title{
Some Perceptions on Research Integrity
}

Sri Lanka Journal of Bio-Medical Informatics 2010;1(4):192-4

DOI: 10.4038/sljbmi.v1i4.2490

This editorial has utilised the content and ideas gleaned from the $2^{\text {nd }}$ World Congress on Research Integrity held in Singapore from the $21^{\text {st }}$ to the $24^{\text {th }}$ of July 2010. However, the author takes full responsibility for the concepts and language used in this article.

The official Singapore Statement on Research Integrity, from that conference, is reproduced elsewhere in this issue of the journal, by kind courtesy and permission from the $2^{\text {nd }}$ World Congress on Research Integrity.

The author also wishes to acknowledge part-sponsorship of his participation at the above Congress by The Postgraduate Institute of Medicine, University of Colombo, Sri Lanka and by A. Baur \& Company, Sri Lanka.

In the world of today, scientific research is the cornerstone of progress. In the specific context of medicine, research is primarily geared towards benefiting suffering humanity. Such being the anticipated paybacks from properly conducted research, it is crucial to realise that the immense value of research rests to a large degree on the integrity of research. The word integrity depicts several meaningful and interrelated connotations. These include honesty, truthfulness, veracity, reliability, uprightness and, by extension, appropriateness of action and responsible behaviour. In the framework of scientific research, all these words have very definite sacrosanct meanings and implications. Especially in bio-medical research, all these words encompass a truly unique quest towards progress and advancement of the said science that would ultimately percolate towards benefiting ill humanity. In such a milieu, the occurrence of breaches in research integrity, academic misbehaviour and misconduct, should be looked at and evaluated using the proverbial fine toothed comb. Given the complexity of the problem, such an initiative is impossible in an editorial of this nature. However, the purpose of this narrative is to draw attention to at least some of the more important aspects of breaches in research integrity.

When many people think of violations of research integrity, most would attribute it to authors. However, it is well documented that breaches in research integrity can be associated not only with authors but also with reviewers, editors, publishers and a whole host of other stakeholders. In the case of authors, such infringements could sometimes be due to genuine and innocent errors such as incompetence and selection of inappropriate methods. However, much more serious and sinister forms of research misconduct are deliberate falsification, fabrication, manipulation and sloppiness. Some of these grave and serious abuses involve tampering with raw data, statistical manoeuvring, image manipulation using PhotoShop computer software ("PhotoShopping"), duplicate publications and frank plagiarism of many different types. All these ultimately add up to intellectual dishonesty and blunt fraud. Sometimes, it is quite amazing to see the lengths to which authors would go to "polish up" 
and "beautify" a scientific paper. In addition, excluding certain authors who have a legitimate right to be cited as authors of papers, provision of "gift authorships" and the use of "ghost authors" are quite serious violations of integrity on the part of researchers.

From the standpoint of research integrity, plagiarism of many different forms and hues is a major problem. There is no doubt that it is a most unacceptable dishonest practice. Genuine quotation of relevant and important information from other articles, scientific or otherwise, is of course eminently permissible and is in fact quite necessary in certain fields such as biomedical sciences. However, there are boundaries that should not be crossed. Plagiarism of entire paragraphs and sometimes even pages, without quotation marks, is the sort of mischievous practice that authors get up to. The current indiscriminate "copy and paste" culture is a practice that should be condemned outright. However, it is perfectly within the boundaries of proper behaviour to cite related work provided they are appropriately acknowledged by proper quotation procedures and by referencing the cited literature.

It is generally argued that quite a few of these misdemeanours are sometimes attributed to the need to publish scientific articles to gain promotions in academic institutions and for career advancement. The frequently used cliché "publish or perish" in certain academic spheres should not be taken as a licence to secure a free hand in all kinds of dishonest practices. In this instance at least, the ultimate goal does not justify the means if all kinds of malpractices are resorted to. It must be stressed that whatever the supposedly mitigating circumstances, violations of research integrity on the part of authors is totally unforgiveable and completely unacceptable.

It may surprise readers that reviewers, editors and even publishers could be involved in serious research misconduct. In the case of reviewers, plagiarism of methods and ideas from manuscripts sent to them for review, data acquisition, non-disclosure of conflicting interests, deliberate negative assessments etc., are just a few of these. In addition, not doing the reviews in time, just performing superficial assessments without attention to detail and generally not taking too much care on the job are some of the other misdemeanours.

In the case of editors, especially selecting certain articles, manipulating articles and references to enhance the Impact Factor, deliberate persecution of certain authors etc., are some of the violations of the trust placed on them for their integrity. It is also the hallowed duty of editors to thoroughly investigate all types of suspicions and allegations that are brought to their notice and not "sweep things under the carpet". As regards integrity, editors should have defined goals and missions, set their paths towards those goals and also motivate others to follow as well. Good editorial practices do not always go hand in hand with one being an excellent researcher or a superlative author. Sound editorial practices additionally need an unwavering commitment to integrity. It is not only authors, reviewers and editors who are sometimes guilty of research malpractices and misconduct but publishers too are involved sometimes in various types of misdemeanours. These are often based on commercial considerations. 
As for the general readership of scientific literature, it is the responsibility of every single one of them to bring to the notice of appropriate authorities, all instances of suspected research misconduct. Very often, even when suspicions are aroused, one takes the easier path of ignoring it and being content to "leave it to others to sort things out". That type of behaviour is abdication of moral responsibility. The editors have a bounden duty on their part to retract papers that have confirmed evidence of research misconduct. In such cases, it is really necessary to publish the reasons for retracting the paper as well. That practice alone may have some effect in functioning as a deterrent. Appropriate action being taken by all concerned including journals and institutions would certainly play a part in reducing the many vagaries of research misconduct.

A synopsis document on broad guidelines for research integrity was formulated at the $2^{\text {nd }}$ World Congress on Research Integrity held in Singapore from the $21^{\text {st }}$ to the $24^{\text {th }}$ of July 2010. That document is reproduced elsewhere in this issue of the journal. Many aspects of publication ethics are also available from the Committee on Publication Ethics (COPE), available from < http://publicationethics.org/>.

Intellectual honesty and transparency in all deeds are mandatory requirements for integrity in scientific research. It naturally follows that it is based on the honesty of all stakeholders, integrity of scientific literature and the veracity of the scientific published public record. There is unlimited freedom for all those who are engaged and involved in scientific research to do the things that have to be done, in a proper manner and within the limits of ethical and moral obligations. It must be realised by all concerned that together with the freedom, responsibility goes hand in hand and freedom without responsibility would be quite meaningless and certainly detrimental to future progress. All of us admire and are ever ready to shower accolades on well conducted, honest and transparent scientific research. The ultimate goal for the scientific community and for the populace at large should be better research for the whole world. It must be reiterated that if that goal is to be universally accomplished, an important and absolutely necessary prerequisite would be to maintain the integrity of the research that is performed and published.

Dr. B.J.C. Perera

Editor-in-Chief 